\title{
BENDABLE VS. ANGULATED DENTAL IMPLANTS: CONSIDERATION OF ELASTIC AND PLASTIC MATERIAL PROPERTIES BASED ON EXPERIMENTAL IMPLANT MATERIAL DATA AND FEA
}

\author{
Tomas Goldmann ${ }^{\mathrm{a}}$, Stefan Ihde ${ }^{\mathrm{b} *}$, Jiri Kuzelka ${ }^{\mathrm{a}}$, Lucie Himmlova ${ }^{\mathrm{c}}$ \\ a Czech Technical University in Prague, Laboratory of Biomechanics, Department of Mechanics, Biomechanics and \\ Mechatronics, Faculty of Mechanical Engineering, Technicka 4, 16667 Prague, Czech Republic \\ ${ }^{b}$ Gommiswald Dental Clinic, Dorfplatz 11, 8737 Gommiswald/SG, Switzerland \\ ${ }^{c}$ Institute of Dental Research $1^{\text {st }}$ Medical Faculty of Charles University and General Medical Hospital, Vinohradska 48, \\ 12021 Prague 2, Czech Republic \\ e-mail:dr.ihde@implant.com
}

Received: May 9, 2008; Accepted: September 29, 2008

Key words: FEM analysis/Basal implants/Bendable implants/Angulated implants

Aim: Bendable and angulated single-piece implants are used alternatively to screwable abutments in two-piece dental implant designs. Though used frequently, data on the stress distribution within such implants are not available and the question whether the bending contributes to fracture resistance has not been addressed.

Methods: We used the method of finite element to identify von Mises stresses and maximum stresses in bent and non-bent but angulated implants. Implants with one (e.g. applicable to screw designs) or two (applicable to basal implants) bending areas were the variables under investigation.

Results: For bends up to 13 degrees we discovered that if there is only one bend, the maximum stress is in the bent area. If two bends are made in two different bending areas, the maximum stresses are distributed between the two and, if either one of the bent areas is machined, there are no residual stresses within the implant body in this area. The maximum stresses are always located near the base-plates. The absolute value of the maximum stress is higher because no residual stresses are available to compensate stresses that stem from loading.

Conclusion: Assuming that all other parameters are equal, bendable (basal) implants show a more even stress distribution along the vertical implant region than identically shaped implants with a machine-angulated area. Bendable basal implants therefore probably resist masticatory forces better than pre-angulated, machined implants, and unbent implants which provide a thin region in the vertical implant area.

\section{INTRODUCTION}

Bendable and angulated single-piece dental implants are used as an alternative to designs which include screwable abutments ${ }^{1}$. The advantage of using one-piece implants is avoidance of gaps between implant body and abutments and to obviate otherwise frequent loosening of abutments or prosthetic screws ${ }^{2}$.

One consequence of jaw bone atrophy may be that no bone is present in those jaw areas where the teeth are to be placed. This creates problems for the implantologist and has led to the desire to perform bone augmentations. Basal implants ${ }^{3}$ overcome this problem in a different manner than cylindrical ones. Their baseplates are anchored in small, native bone areas often far from the actual clinical tooth, i.e. distant in a vertical and/or sagittal and/or horizontal direction. Although clinical experience has led to the development of a variety of basal implant designs, the surgical situation may demand variable positioning of the interconnection implant part (screw hole or cementing post). For this reason, the surgeon has to bend the vertical part of the implants before or after the insertion, in order to allow easy prosthetic access of the connection area.

Although bending of implant necks has been used in crestal implantology for a long time, no literature was found on the effects of bending on the development of residual stresses within the implant and alteration of the stress distribution during loading. The description of the effects takes into account that plastic bending affects the internal structure of the implant body because the described deformations exceed the yield limits of the material.

We have developed a model that takes into account the following:

- the process of bending an inserted implant is assumed to be harmless to the bone if the resulting forces on the bone interface remain within the range of elastic deformation of the bone, thus creating no or only a limited number of cracks or micro-cracks

- the forces must be within a range that can be applied inside the oral cavity by the surgeon.

In previous articles we created models to describe the interaction between bone and basal implants using the 
method of finite element analysis. Thereby we proposed a novel approach for modelling different interactions between cortical and cancellous bone $e^{4,5}$. This approach has been used for describing different stages of osseointegration and remineralization. In daily clinical practice as well as during previous computations, the problem of stress distribution within dental implant bodies undergoing intra-operative bending vs. implant bodies which have been machined in angulated designs out of one piece of metal became of interest.

\section{MATERIAL AND METHODS}

The understanding of dental implants mechanical behavior during bending process comes from clinical practice findings, where the problem of the durability prediction during implantation procedure arises. A set of finite element models describing possible bending cases formed at implantation was chosen as a suitable method. However material experiment takes also part in this study (Titanium Grade 2), where results are used to improve material model.

As a first step to obtaining reliable results, we performed a tensile testing experiment on a Titanium Grade 2 specimen. In this way the material model is extremely precise over a wide range of loading. We used rod specimens for this experiment with a total length of $190 \mathrm{~mm}$ and a diameter of $10 \mathrm{~mm}$. The specimen was connected to the testing machine through M16 threads at both ends (Fig. 1a, b). We tested 5 specimens and the results were extremely similar. Average values and standard deviations (tensile yield strength $\sigma_{\mathrm{k}}=326 \pm 11.40$ and ultimate tensile yield strength $\sigma_{\mathrm{p}}=492.6 \pm 2.41$ ) were used for calculation.

Our model is based on the stress-strain-curve shown in Fig. 2. This figure shows three lines (0-A; A-B, B-C), with the line $0-\mathrm{A}$ corresponding to the elastic material behaviour, lines A-B and B-C approximate the properties of the material in the plastic zone. The line decreases on the right side of $\mathrm{C}$ since the cross sectional area of the specimen diminishes during the experiment.

Since we found a considerable difference in repeated measurements and the available data in the literature ${ }^{6}$ (Fig. 3), we decided to trust our own measurements and used the model obtained values from our material tests. In the above reference ${ }^{6}$ the ultimate strength is reported to be higher than the maximum value of the yield strength, which we consider unusual. Also for this reason we decided to perform a test of our own.

As a second step to approach the questions arising from angulated or bendable dental implants we chose to observe the stresses within the implants and the influence of the bending action, without considering the stresses at the interface to bone. Thus we modeled through the method of finite elements several bending situations for basal implants with one baseplate. The finite element mesh of the mandible was created in the system ABAQUS 6.6-5 (Abaqus Inc., Providence, RI 02909-2499, USA) by the C3D4 element type. The ABAQUS system was also used for all remaining pre-processing, computational and result evaluation works. The practically usable cross sectional diameter of the bending area for a dental implant (made from c.p. Titanium Grade 2) can only range from $1.7 \mathrm{~mm}$ to $2.0 \mathrm{~mm}$. Smaller diameters should not be considered because they are prone to fracture, while implants with higher diameters do not allow bending inside the oral cavity.

For our calculations, the shaft thickness was assumed to be $1.95 \mathrm{~mm}$ and the resulting upper and lower bending angles after plastic deformation and elastic relapse were each 13 degrees. The vertical implant part had no contact with bone and the baseplates were rigidly anchored. Four cases of bending or double bending of the vertical implant part were calculated (Fig. 4).

Since we found in preliminary calculations ${ }^{4}$ that only very little stress occurs within the baseplates in these implant designs, we were able to simplify the model into a plane symmetrical part of implant and leave out the desk (i.e. the fixating baseplate; Fig. 5). In this way our findings can also be applied to the crestal implant designs, i.e. screws. However in screw designs only the upper bending area is of clinical use.

The analysis of each case (Fig. 4) considers a sequence of mechanical actions imposed on the implants:

- Step 1: before any bending, the implant was fixed below the particular bending area

- Step 2: the implant part above the notch was then bent, applying kinematical load. The initial bending angle was 15 degrees

- Step 3: immediately after releasing the necessary loading, an elastic relapse was assumed to occur, resulting in a total bending angle of 13 degrees

- Step 4: after the bending, stresses within the pre-bent implants under loading conditions were calculated. Note that von Mises stresses and main stresses were calculated and displayed.

- the actual loading was calculated in two ways:

1. Main stresses and von Mises stresses were calculated for all four cases, assuming static loading of $450 \mathrm{~N}$, with the load being applied perpendicular to the crestal abutment surface.

2. Results were compared to implants which had not been bent but manufactured by $\mathrm{CNC} /$ machining into exactly the same angulation. These implants showed no residual stresses inside the area of angulation because they have not been bent. A reduced force of only $390 \mathrm{~N}$ was applied in all cases where the upper bending area was bent. We had to use a lower force because the computation would be inaccurate if the value of stress determined at point $\mathrm{C}$ (Fig. 2) was reached or exceeded.

In the cases of two bendings (case 3 and 4, Fig. 4), we assumed that the results of the second bending procedure did not affect the first bending.

The implant material, considered in this model, is c.p. Titanium grade 2, ISO 5832-2:1993. Elastic properties are described by Young's modulus of elasticity $E=$ $1.05 e 5 \mathrm{MPa}$ and Poisson's ratio $v=0.37$. The plastic prop- 

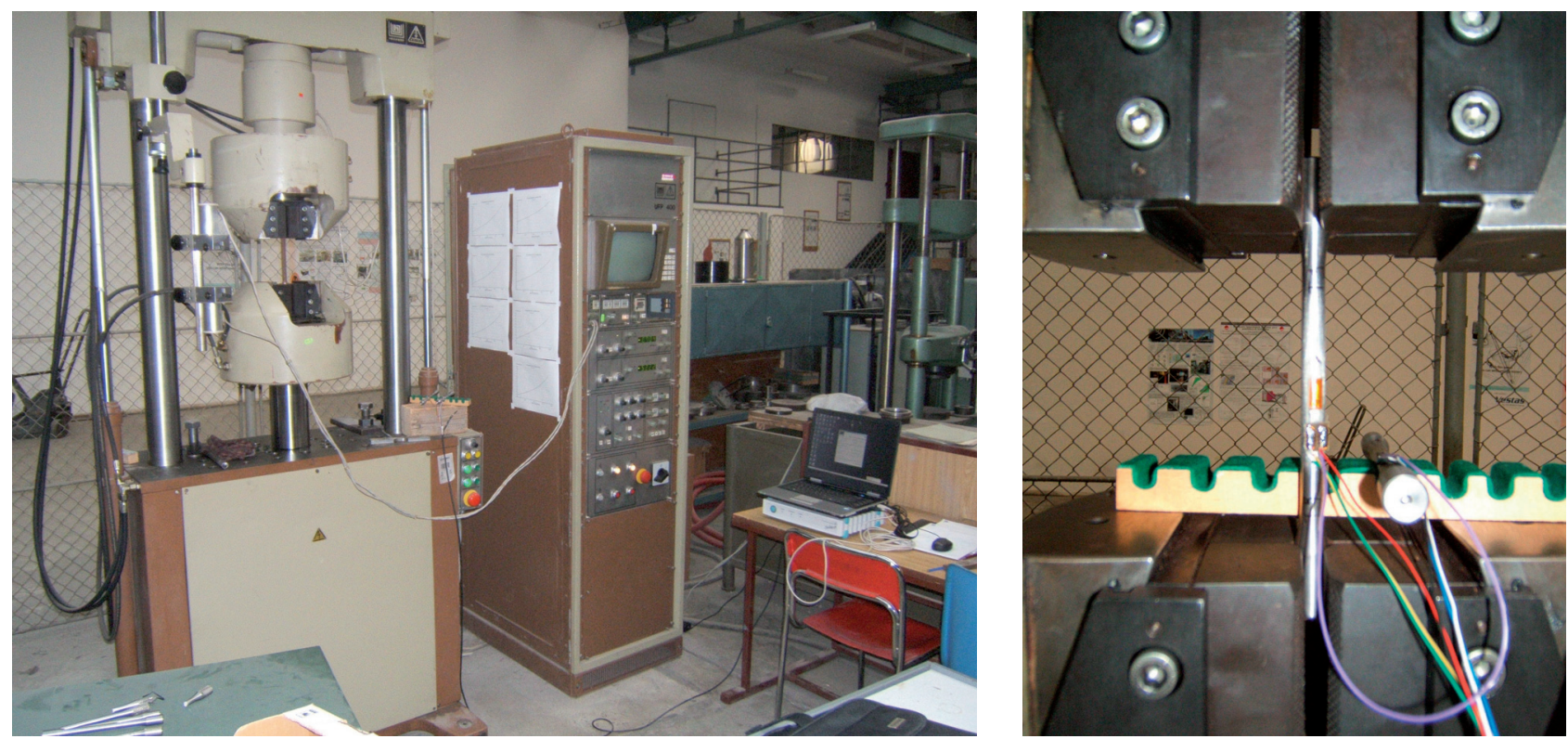

Fig. 1a, b. Heckert VFP400 tensile testing device used for our testing, with the test specimen made from "Titanium Grade 2".

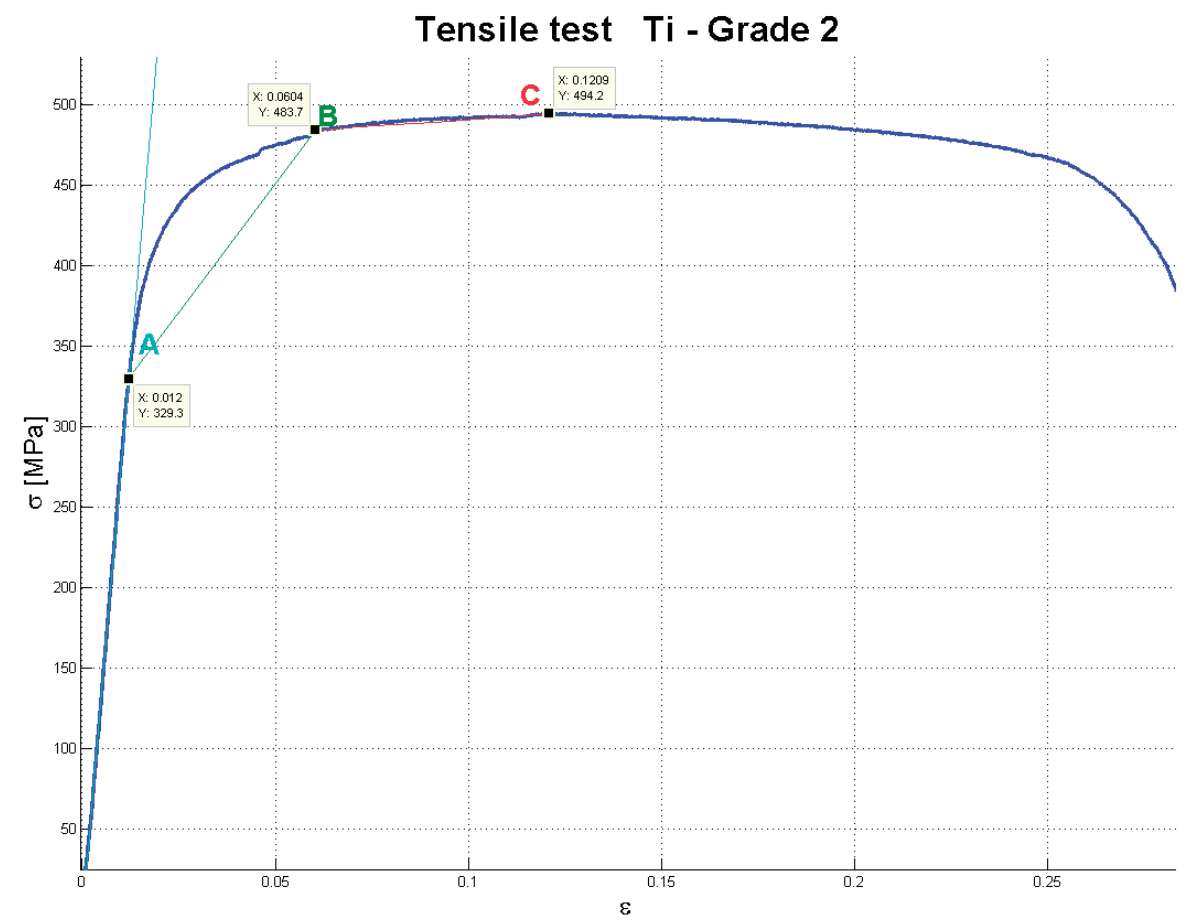

Fig. 2. Results of tensile testing of the Ti Grade 2 rod. A (yield point): $x=0,0123$; $\mathrm{y}=329,3$, determining the end of the elastic zone; $\mathrm{B}$ (a second defined point for our material model): $\mathrm{x}=0,0604 ; \mathrm{y}=483,7 ; \mathrm{C}$ (endpoint of our model definition): $\mathrm{x}=0,01209 ; \mathrm{y}=494,2$.

erties are taken from our material test described above where the stress-strain curve was approximated by three points (A, B, C). The above-mentioned material properties are shown in Figure 2 for illustration. The material in the plastic stage is modelled as incompressible $(v=0.5)$. The material is assumed to change its material properties isotropically during plastic deformation but these changes have not been quantified to our knowledge.

\section{RESULTS}

\section{Bent implants}

Examples of Von Mises stress distribution [MPa] in range from the yield stress to the failure stress at the moment of maximal bend (Step $2-15^{\circ}$ bending), the release of the kinematical loading (Step $3-13^{\circ}$ bending) or final loading by the force (Step $4-450 \mathrm{~N}$ or $390 \mathrm{~N}$ 


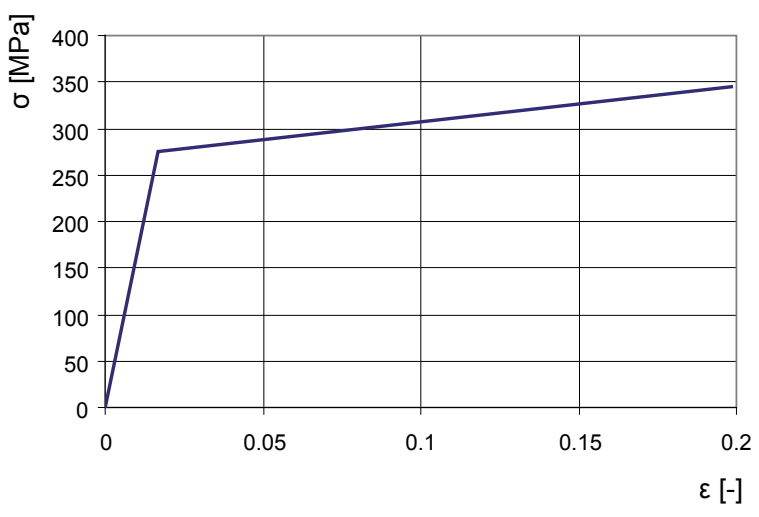

Fig. 3. The range of elastic deformation of Titanium Grade 2 reaches up to approx. $275 \mathrm{MPa}$ (lower value before mechanical working while the almost horizontal, upper part of the line represents plastic material behaviour).

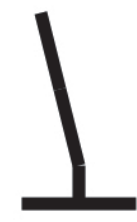

1

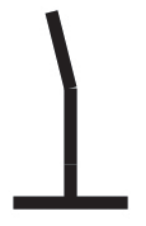

2

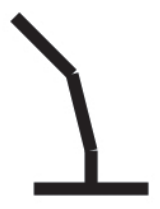

3

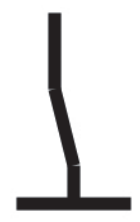

4
Fig. 4. The four cases of bent or double-bent implants calculated in this publication are shown above. Two notches are integral part of the vertical part of the basal implants under examination. The upper notch is positioned underneath the prosthetic connection parts, the lower notch is found slightly above the baseplate.

loading) for all four cases are shown in Figs. 6 - 15. The coloured range corresponds to the plastic zone. Residual von Mises stress distribution is distinguished from stresses under loading and from stresses during the actual bending phase. This value is slightly higher than failure stress in some cases. Moreover the tensile stress is positive and thus there is a serious risk for cracks.

Figures 6, 7 and 8 show von Mises stress distribution for Case 1. Figure 6 displays the situation after kinematical $15^{\circ}$ bending (Step 2); the maximum stress here is $495 \mathrm{MPa}$. This maximal stress surprisingly increases after the release of the bending load (Step 3) up to $530 \mathrm{MPa}$ (Fig. 7) which is caused by the residual stress. The stress decreases again $(515 \mathrm{MPa})$ after loading the implant by a force of $450 \mathrm{~N}$ (Fig. 8). This shows that loading of the implant after bending affects the residual stress and a more balanced stress distribution is the result.

A more or less similar situation occurs again for remaining Cases 2, 3 and 4. The Figure 9 displays von Mises stress distribution at a maximum load of 390 N (Step 4) after one single bend had been applied to the upper bending area (Case 2). Note that there is also a deformation in the area of the lower bending zone stemming from the loading. (Maximum stress is $528 \mathrm{MPa}$ but note that the loading force is different from Fig. 8). Figure 10 shows the residual stresses (Max stress is $530 \mathrm{MPa}$ ) in the vertical implant after two bends had been released (Case3, Step 3) and Figure 11 shows the stress increase (up to $591 \mathrm{MPa}$ ) after loading the implant by a force of $390 \mathrm{~N}$ (Case 3, Step 4). Case 3 is the only case where the loading in Step 4 causes no residual stresses and produces the most balanced stress distribution. Figures 12 and 13 describe Steps 3 and 4 for Case 4. The greater stress distribution (530 $\mathrm{MPa}$ ) is again in the state of the bend release (Step 3) than in the state of final loading (Step 4 - maximum stress is $519 \mathrm{MPa}$ ).

\section{Unbent implants, machined with one angulation in the vertical implant shaft}

If the internal structure of the vertical implant part is homogenous, no residual stresses are present in the (machined) upper angulation area. In this case off-axis loading will lead to a peak stress near the baseplate. This is also true for cases without a circular notch (not shown here): in these cases the peak stresses may be displaced nearer to the baseplate (Fig. 14).

The same applies to basal implants if two angulations are machined into the vertical part (Fig. 15).

Our findings may be summarized as follows:

- if there is only one bending carried out, the maximum stresses are always found in the bent area

- if two bends are carried out, the maximum stresses are distributed between the two bent areas

- if only one of the bent area is machined, there are no residual stresses within the implant body in this area and maximum stresses are always located near the baseplates. The absolute value of the maximum stresses is higher because no residual stresses are available to compensate stresses that stem from loading.

\section{DISCUSSION}

The method of the finite element model is frequently used in evaluating the design of dental implants ${ }^{7,8}$. Most studies evaluate either the stresses imposed on the bone $\mathrm{e}^{9,10}$ or on the prosthetic workpieces ${ }^{11,12}$. We found one study showing that staggered implant placement produces similar stresses to straight placement ${ }^{13}$. No evaluations of bendable implant designs were found in the existing literature and hence the latter provides only limited grounds for comparison with the results of others.

It is possible to apply bends to the vertical implant part more than 13 degrees. However, we chose the maxi- 

on experimental implant material data and FEA
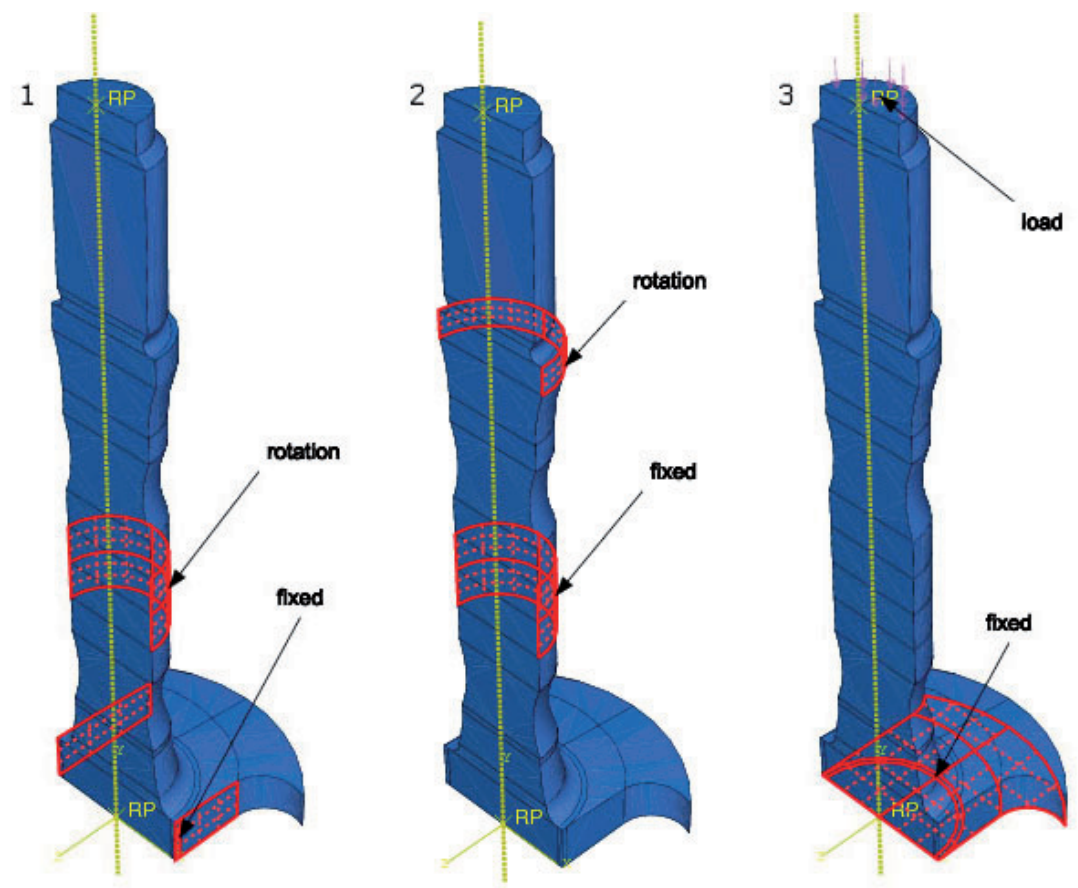

Fig. 5. Plane symmetrical model of the implant, showing the areas of fixation. The term "rotation" of the ABAQUS terminology (www.abaqus.com) describes the bent(-over) part of the vertical implant section.
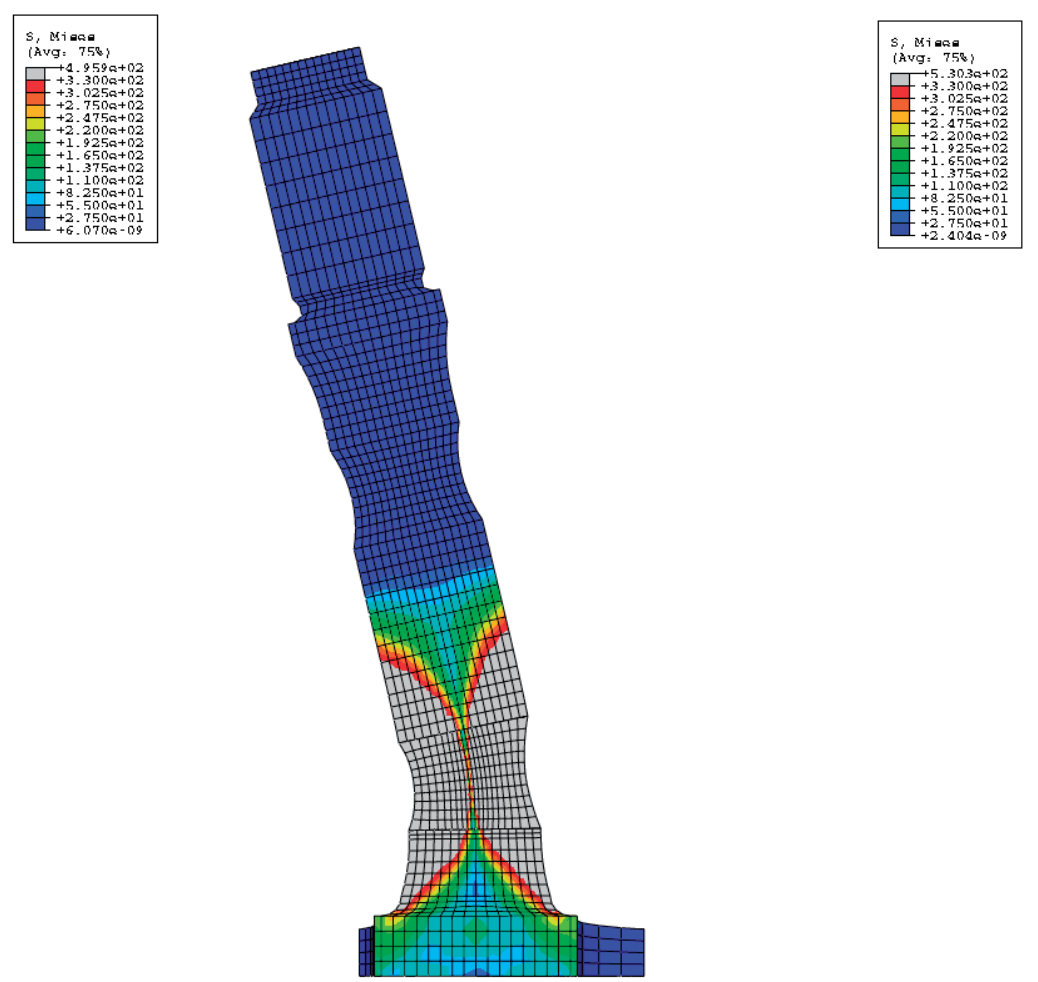

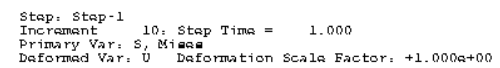

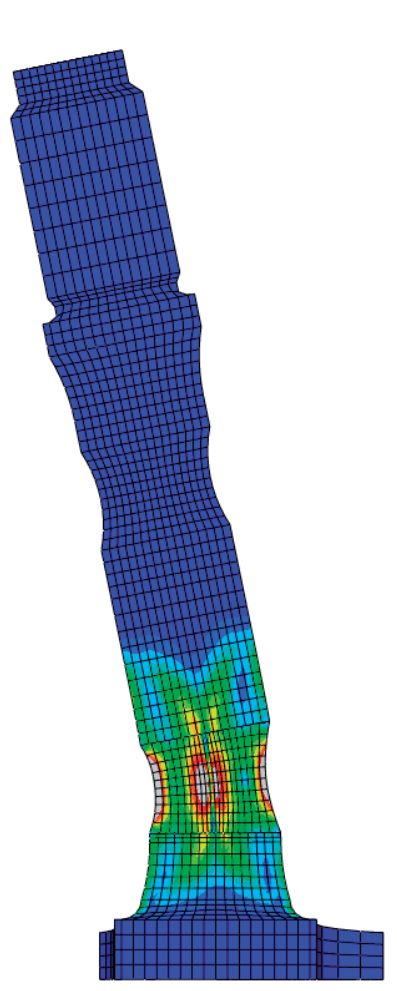

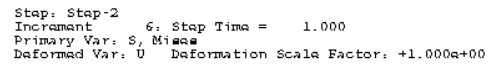

Fig. 6. Case 1, Step 2 von Mises stress distribution in $\mathrm{MPa}$ (Maximum stress is $495 \mathrm{MPa}$ ).
Fig. 7. Case 1, Step 3 von Mises stress distribution in $\mathrm{MPa}$ (Maximum stress is $530 \mathrm{MPa}$ ). 

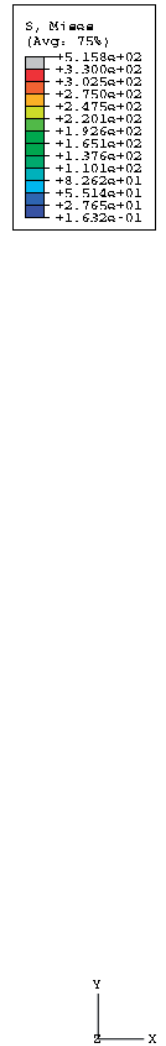

Stap: Stap -3

Incramant -3
Primary Var: S, S: Stap Time $=1.000$

Fig. 8. Case 1, Step 4 von Mises stress distribution in $\mathrm{MPa}$ (Maximum stress is $515 \mathrm{MPa}$ ).
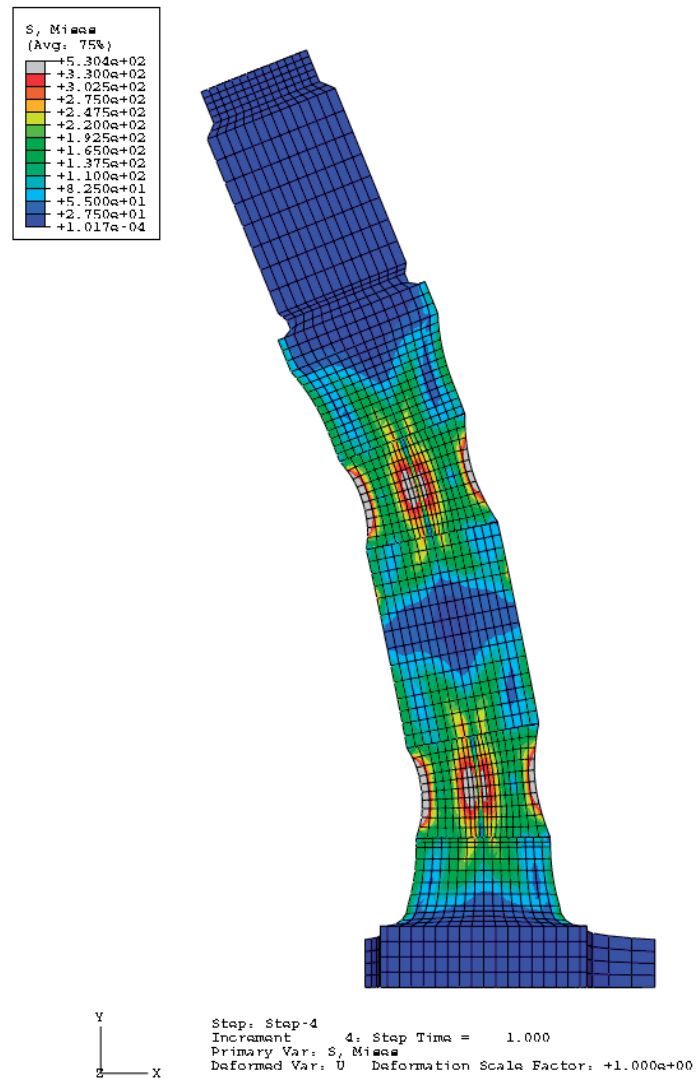

Fig. 10. Case 3, Step 3 von Mises stress distribution in $\mathrm{MPa}$ (Maximum stress is $530 \mathrm{MPa}$ ).
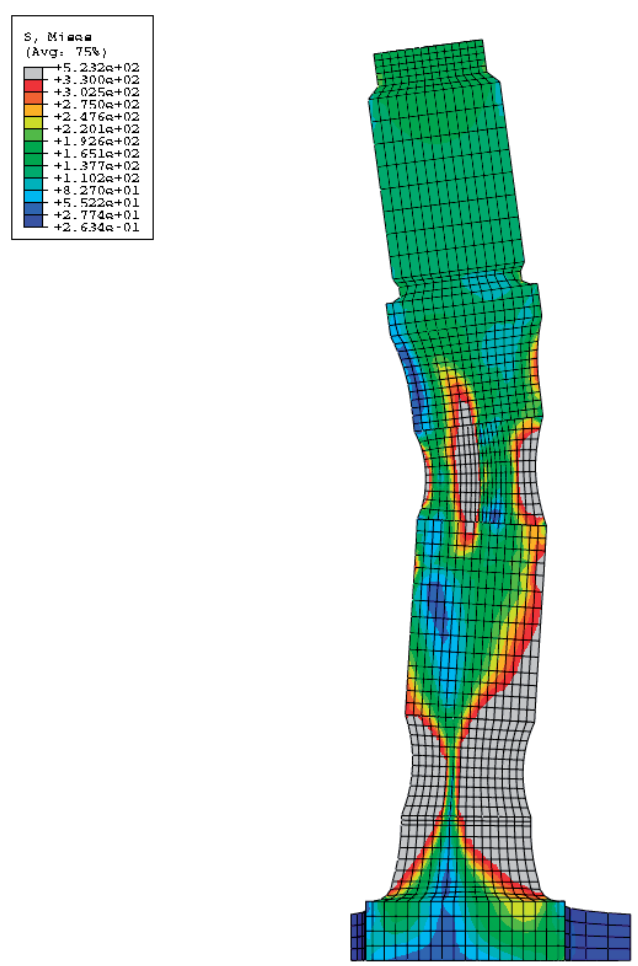

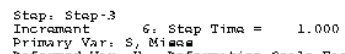

Fig. 9. Case 2, Step 4 von Mises stress distribution in $\mathrm{MPa}$ (Maximum stress is $528 \mathrm{MPa}$ ).
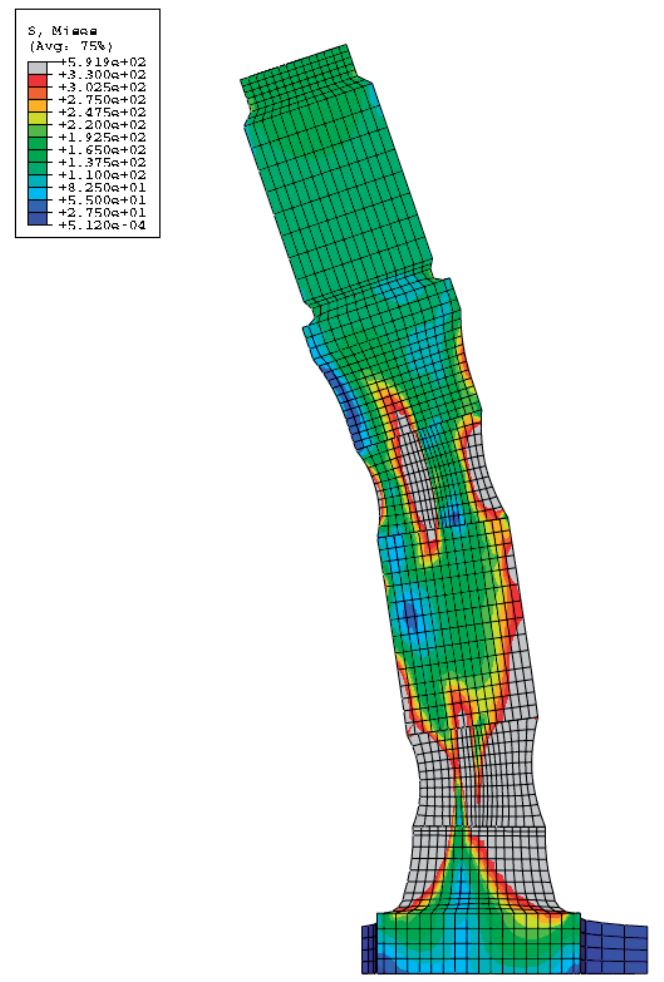

Stap: Stap -5
Incremant
Priman
Drestap Tima $=1.000$

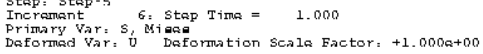

Fig. 11. Case 3, Step 4 von Mises stress distribution in $\mathrm{MPa}$ (Maximum stress is $591 \mathrm{MPa}$ ). 

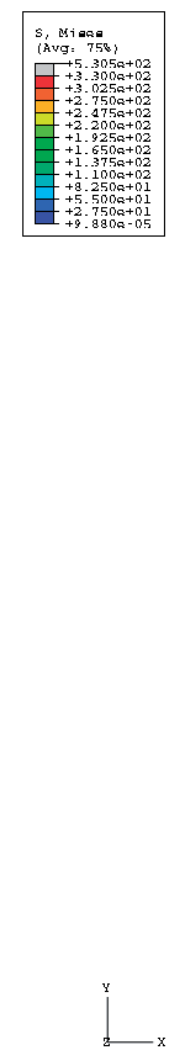

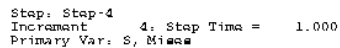

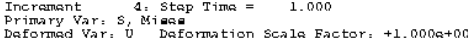

Fig. 12. Case 4, Step 3 von Mises stress distribution in $\mathrm{MPa}$ (Maximum stress is $530 \mathrm{MPa}$ ).

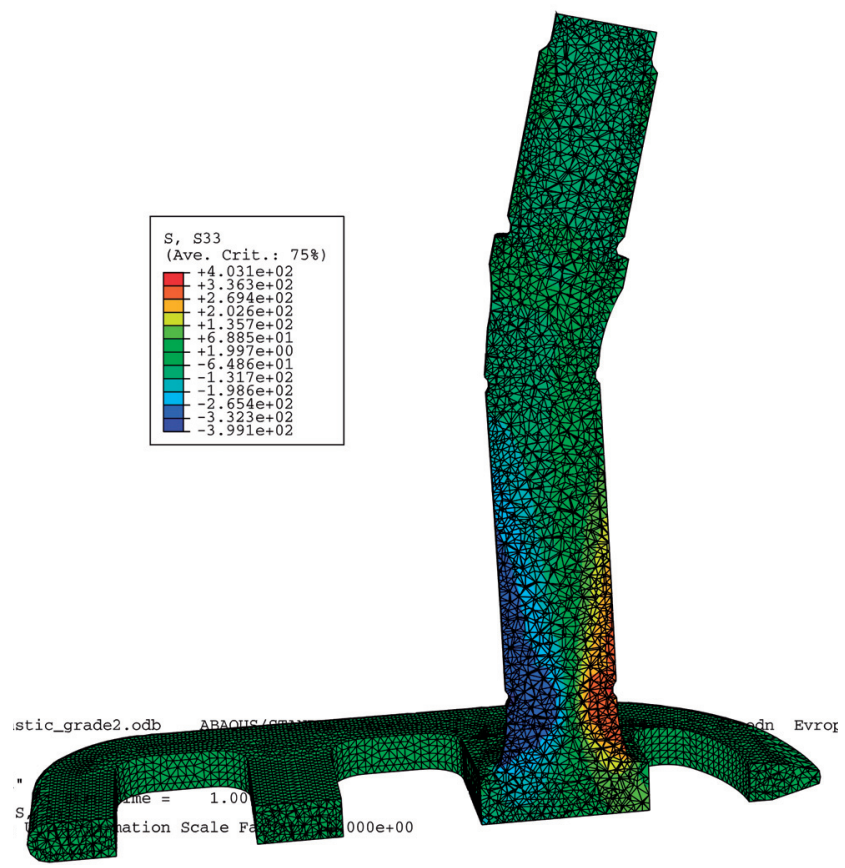

Fig. 14. Von Mises stress distribution within a basal implant with a machined upper angulation area (in $\mathrm{MPa}$ ). The same applies to basal implants if two angulations have been machined into the vertical part of Fig. 15.
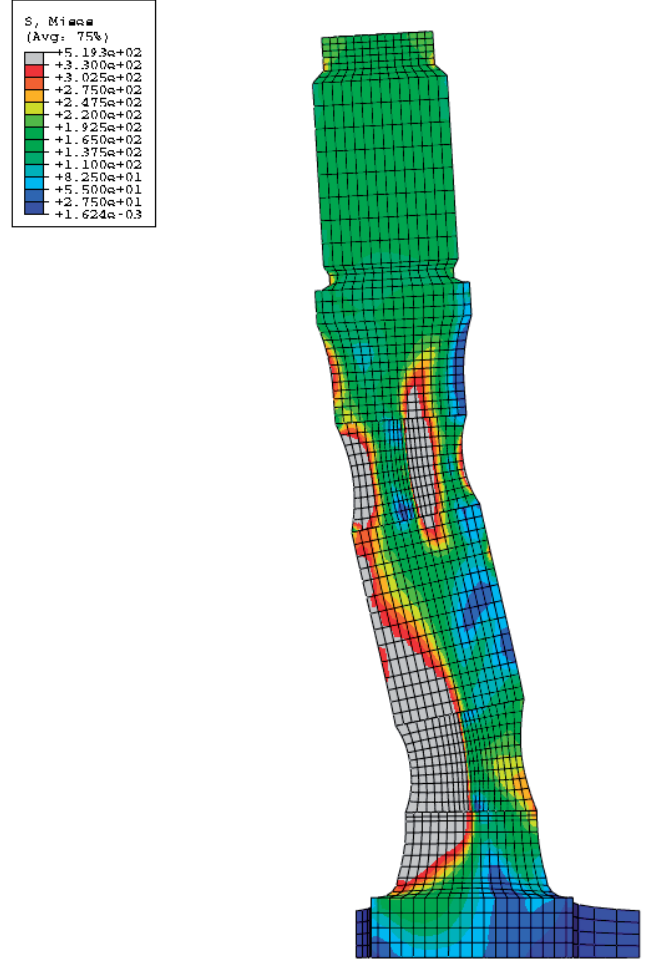

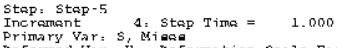

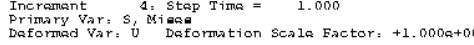

Fig. 13. Case 4, Step 4 von Mises stress distribution in MPa (Maximum stress is $519 \mathrm{MPa}$ ).
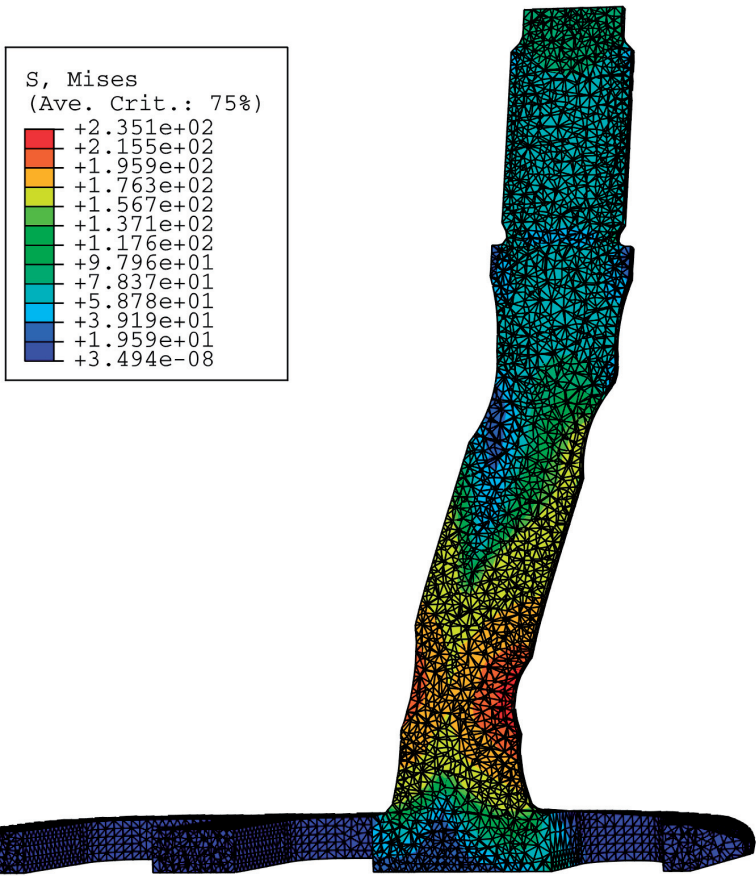

Fig. 15. Von Mises stresses within a basal implant with two machined angulations in the vertical implant portion. 
mum degree of bending (after Step 2) since when we tried to calculate with larger bending angles, the computation failed. In our model the situation changed so dramatically with bends of more than 13 degrees, that any calculation was unfeasible. Larger bends are clinically possible and also performed in daily clinical practice but our model did not allow us to compute them. We did not calculate machined pre-angulated implants with a greater angulation because the bendable counterpart was not available for comparison in our model.

The highest stresses were found when exerting a maximum bending of 15 degrees. This stress consists of elastic and plastic components. After the elastic relapse to a final bending of 13 degrees, the plastic deformation and the residual stresses remain, while the elastic stresses vanish. The strong internal forces from plastic deformation are not visible. However, they affect the stress distribution during subsequent loading. Our findings are applicable to a wide range of materials. However a large number of materials will not allow bending under the conditions applicable inside the oral cavity. Interestingly the different types of bending (Cases 1-4) do not alter the maximum stresses dramatically.

One of the weaknesses of this study is the fact, that the interface to bone was not considered. Hence the results are valid only for basal implants immediately after the implant placement, in cases where the implant is placed in highly mineralized, cortical bone providing a rigid fixation of the baseplate with no contact between the vertical implant part and the bone. The situation during the dual process of osseointegration of a basal implant has been described for non-angulated, unbent implants and oblique loading ${ }^{5}$. However, calculations for angulated or bent osseointegrated implants and the determination of forces acting on the bone while implants are bent, remain to be performed in future. Also the effect of cyclic loading on the bending areas was not calculated, since fatigue computations are not routinely performed in finite element analysis.

When transferring the results from basal to screw-type implants and the clinical reality, we must keep in mind that the insertion of bendable implants into more mineralized bone (Types D1, D2) may lead to extremely high torque forces, which exceed the forces during bending by far. These forces may lead to fracture of the bending area, although the implant may have been well able to withstand the bending and the following cyclic masticatory loading without damage, in the case that the implant had only been inserted without damage.

\section{CONCLUSIONS}

Assuming that all other parameters are equal, bendable basal dental implants show a more even stress distribution along the vertical implant part compared to identical angulated implants. Bent implants of this type probably therefore resist masticatory forces better than angulated implants. Our results can be transferred to screw implants with thin, bendable necks: those implants should undergo bending whenever they are used, in order to improve material properties through residual stresses and thereby increase mechanical resistance in the bending area.

\section{ACKNOWLEDGEMENTS}

This study was funded by the Czech Ministry of Education project No. MSM 6840770012 and by the Grant Agency of the Czech Republic under project No. 106/06/0849.

\section{REFERENCES}

1. Bauer E. Dentalimplantat EP 0320740 B1; 5.12.1988.

2. Binon PP. Evaluation of the effectiveness of a technique to prevent screw loosening. J Prosthet Dent 1998; 42:71-89.

3. Scortecci G, Misch CE, Benner K. Implants and Restorative Dentistry. 2000, Martin Dunitz,: Martin Dunitz, London; 2000.

4. Ihde S, Goldman T, Himmlova L, Aleksic Z. The Use of Finite Element Analysis to Model Bone-Basal Implant Contact. Oral Surg Oral Med Oral Pathol Oral Radiol Endod. 2008; 106(1):39-48.

5. Ihde S, Goldman T, Himmlova L, Aleksic Z, Kuzelka J. Implementation of contact definitions calculated by FEA to describe the healing process of basal implants. Biomed Pap Med Fac Univ Palacky Olomouc Czech Repub 2008; 152(1):169-173.

6. Titanium grade 2 composition, available from: http://www.matweb. com/search/SpecificMaterial.asp?bassnum=MTU020.

7. DeTolla DH, Andreana S, Patra A, Buhite R, Comella B. Role of the finite element model in dental implants. J Oral Implantol 2000; 26(2):77-81.

8. Geng JP, Tan KB, Liu GR. Application of finite element analysis in implant dentistry: a review of the literature. J Prosthet Dent 2001 Jun; 85(6):585-98.

9. Geng JP, Xu W, Tan KBC, Liu GR. Finite Element Analysis of an Osseointegrated Stepped Screw Dental Implant. J Oral Implantol 2004; 30:223-33.

10. Holmgren EP, Seckinger RJ, Kilgren LM, Mante F. Evaluating Parameters of Osseointegrated Dental Implants using finite element Analysis - a two-dimensional Comparative study examining the effects of implant diameter, implant shape, and load direction. J Oral Implantol 1998; 22(2):80-8.

11. Ciftci Y, Canay S. Stress distribution on the metal framework of the implant supported fixed prosthesis using different veneering materials. Int J Prosthod 2001; 14(5):406-11.

12. Geramy A, Morgano SM. Finite element analysis of three designs of an implant-supported molar crown. J Prosthet Dent 2004; 92(5):434-40.

13. Kivanc A, Haldun I. Finite element analysis of the influence of staggered versus straight placement of dental implants. Int J Oral Maxillofac Impl 2001; 16(5):722-30. 